\title{
Patientensicherheit: Lehren der Luftfahrt für den Gesetzgeber
}

\section{Hanspeter Kuhn}

Fürsprecher und bis 31.3.2018 Abteilungsleiter Rechtsdienst sowie stv. Generalsekretär der FMH

\author{
Zur Motion 17.3974 «Schadensprävention und Umgang mit Schäden bei medizini- \\ schen Behandlungen» der nationalrätlichen Kommission für soziale Sicherheit \\ und Gesundheit (SGK-N).
}

Die am 3. November 2017 eingereichte Motion der SGK-N verlangt die «Stärkung und flächendeckende Implementierung einer Sicherheits- und Fehler-Lernkultur verbunden mit Regressmöglichkeiten und der Erleichterung der Beweissituation durch Verbesserung der Behandlungstransparenz insbesondere bei fehlerhaften Eingriffen.»

Voraussetzung für eine erfolgreiche Sicherheits- und Fehler-Lernkultur ist die Sicherheit der Mitarbeitenden, dass sie im Zusammenhang mit gemeldeten kritischen Ereignissen (critical incidents) keine Sanktion befürchten müssen. Die gleichzeitige Regressmöglichkeit und Erleichterung der Beweissituation stehen im Widerspruch dazu. Beides aufs Mal ist nicht zu haben.

\section{Vertraulichkeit ist Voraussetzung}

Linda J. Connell, die Direktorin des NASA Aviation Safety Reporting System der US-Zivilluftfahrt (ASRS), hat schon im Februar 2000 anlässlich der Anhörung vor dem Ausschuss des US-Repräsentantenhauses [1] darauf hingewiesen, dass der Vertraulichkeitsschutz und der Schutz vor Verfolgung entscheidende Erfolgsfaktoren für eine Fehlermeldekultur sind:

"The reports sent to the ASRS are held in strict confidence. More than 470000 reports have been submitted since the program's beginning without a single reporter's identity being revealed. The ASRS removes all personal names and other potentially identifying information before entering reports into its database [...] The reporters to ASRS are guaranteed limited immunity by the FAA. This means that the FAA will not use, nor will NASA provide, information that has been filed with the ASRS in an enforcement action, and will waive fines and penalties for unintentional violations of Federal Aviation Regulations, as long as violations are reported within 10 days."

\section{"Critical incidents» statt "accidents» als Lernquelle}

«Der Mensch ist jede Stunde des Tages zum Mindesten ein Mal unvorsichtig. Das liegt in seiner Natur. [...] Die Natur des Menschen ändert sich aber nicht mit seinem Eintritt in den Fabrikraum, mit seiner Anstellung als Eisenbahnkondukteur.» Dies schrieb Nationalrat Ludwig Forrer, der Vater der Schweizerischen Unfallversicherung, im Jahr 1889! [2]

Sicherheitskultur baut deshalb auf der Fehlbarkeit des Menschen auf. Dabei muss sich das Fehlermeldesystem auf Beinahe-Schäden beschränken, weil es nicht möglich wäre, die Immunität im Zusammenhang mit ernsthaften Schadensereignissen aufrecht zu erhalten. "Do not report aircraft accidents on this form", steht deshalb zuoberst auf dem CIRS-Formular des Aviation Safety Reporting System der NASA für die amerikanische Zivilluftfahrt. Denn: "Accidents and criminal activities are not included in the ASRS-program and should not be submitted to NASA."

Linda Connell wies auf die zentrale Bedeutung des Vertraulichkeitsschutzes hin:

"But all countries are very aware of their survivability in relation to confidentiality. As an example to all systems, one country's first system was completely destroyed due to lack of reporting after a breach of a reporter's identity."

Der US-Kongress hat 2005 in der Patient Safety Act die Vertraulichkeit [3] von Meldungen im Rahmen von CIRS- und anderen Qualitätssicherungsprogrammen sichergestellt. Er hat damit umgesetzt, was die American Medical Association 2002 vorgeschlagen hatte:

"Congress can help create a culture of safety by allowing medical professionals to convene to discuss patient safety problems and potential solutions without having their discussions, findings, or recommendations become 
the basis for class action or other lawsuits. If the fear of litigation continues to pervade efforts to improve patient safety and quality, our transformation into a culture of safety on behalf of our patients may never be fully realized [4]."

Der Bericht des BAG «Patientenrechte und Patientenpartizipation in der Schweiz» vom 24. Juni 2015 [5] weist auf das Gutachten von Bernhard Rütsche, Nadja d'Amico und Lea Schläpfer von 2013 hin [6]:

\section{Vertraulichkeitsschutz und der Schutz vor Verfolgung sind entscheidende Erfolgsfaktoren für eine Fehlermeldekultur (Linda J. Connell, Direktorin des NASA Aviation Safety Reporting System der US-Zivilluftfahrt)}

«Fehlermeldesysteme, in allen untersuchten Ländern verbreitet, sind den Experten zufolge von entscheidender Bedeutung. Eine staatliche Regelung der vielerorts bereits bestehenden Systeme könnte deren Funktionsfähigkeit etwa durch die Statuierung von Beweisverwertungsverboten in Zivil-, Straf-, und Verwaltungsverfahren fördern.»

Das Gutachten von Rütsche et al verweist u.a. auf die Regelung in Dänemark von 2007, die Sanktionsfreiheit sicherstellt [7].

\section{Gesetzesvorschlag seit 2001 vorliegend}

Die FMH hat bereits 2001 [8], sodann 2003 [9] und erneut 2009[10] auf die Notwendigkeit einer gesetzlichen Regelung für die Schweiz hingewiesen und bereits 2001 eine Ergänzung z.B. eines Art. 80a des Bundesgesetzes über den Allgemeinen Teil des Sozialversicherungsrechts ATSG vorgeschlagen:

«Und die Schweiz? Die bei uns laufenden Critical-Incident-Reporting-Programme sind gezwungenermassen alle auf die bloss vertragliche bzw. statutarische $\mathrm{Zu}$ sicherung gebaut, Informationen über den Melder vertraulich zu halten. Bisher sind keine Lecks bekannt geworden. Mangels ausdrücklicher gesetzlicher Schutzgarantien sind aber auch unsere Programme verletzlich gegenüber richterlichen Zeugenvorladungen und Akteneditionsverfügungen unter Androhung von Strafe im Verweigerungsfall. Wie in den USA gilt auch in der Schweiz: Dies kann nicht die Lösung sein. 〈Legal protections are the only possible way ...) [11]» In der Schweiz ist nun genau dies eingetreten: Im Kanton Tessin hat die Staatsanwaltschaft 2016 eine CIRSMeldung beschlagnahmt, und das Bundesgericht hat ihre Verwendung im Strafverfahren erlaubt [12]. Schon im Vorfeld hat der CIRRNET-Ausschuss der Stiftung Patientensicherheit Schweiz festgehalten:
"Grundgedanke von Fehlermeldesystemen (CIRS) ist, dass durch das Erfassen und Analysieren kritischer Ereignisse Erkenntnisse abgeleitet werden können, die dazu dienen, daraus zu lernen und denselben Fehler in Zukunft zu vermeiden. Sie basieren dabei auf wesentlichen Merkmalen wie der Vertraulichkeit, Freiwilligkeit, Sanktionsfreiheit und der Identifikation und Bearbeitung von risikorelevanten Schwachstellen im System. (...)

Wir befürchten, dass die Verwendung von CIRS-Meldungen für die juristische Aufarbeitung von potentiellen Fehlern unabsehbare Folgen für die Sicherheitsund Lernkultur im Gesundheitswesen haben wird. Schon jetzt wird ein Rückgang des Vertrauens der Mitarbeitenden in das CIRS in den Akutspitälern des Kantons Tessin berichtet. Wir gehen von weiteren negativen Folgen für die Sicherheits- und Lernkultur und schlussendlich für die Patientensicherheit in der ganzen Schweiz aus. Damit würde der in den letzten Jahren erreichten Kulturveränderung im offenen Umgang mit Fehlern ein immenser Schaden zugefügt. Damit schadet man schlussendlich dem Patienten. Die Stiftung Patientensicherheit Schweiz und der CIRRNET-Ausschuss sprechen sich klar dafür aus, dass Einträge in Fehlermeldesystemen nicht durch die Staatsanwaltschaft konfisziert werden, da sie auf der freiwilligen Meldebereitschaft der Mitarbeitenden beruhen und einem anderen Zweck dienen [13].»

\section{Ohne gesetzlichen Vertraulichkeitsschutz keine Sicherheit(skultur)}

Was ist schief gegangen? Gemäss Bundesgericht erfolgte die fragliche Meldung ins Qualypoint-CIRS-System des Spitals im Zusammenhang mit dem Suizid eines Patienten. Das Spital hat im Zusammenhang mit der Beschlagnahmeverfügung der Staatsanwaltschaft keine Siegelung der Akten verlangt. Soweit der bedauerliche Einzelfall.

\section{Wenn Behörden Zugriff auf CIRS-Meldungen} haben, schwindet das Vertrauen der Gesundheitsfachleute in ein Fehlermeldesystem.

Doch das zentrale Problem ist, dass die Schweiz im Unterschied zu anderen Ländern bisher keinen gesetzlichen Vertraulichkeitsschutz für Meldesysteme von kritischen Ereignissen und Qualitätssicherungsprogramme hat. Das heisst: Die Identität von Personen, die critical incidents rapportieren oder über die rapportiert wird, ist gesetzlich nicht geschützt.

Ich hoffe, dass das Parlament nach dem Fall im Tessin nun rasch von den Erfahrungen anderer Länder profi- 
tiert und die seit 2001 bekannte gesetzgeberische Lücke zügig schliesst. Es wird damit einen wichtigen Schritt zur Förderung des Vertrauens aller Gesundheitsfachleute in die Institutionen unseres Landes tun.

\section{Literatur}

1 Linda J. Connell, Statement before the US Subcommittee on Health, Committee on Ways and Means, House of Representatives, February 10, 2000

2 Ludwig R. Forrer, Denkschriftt über die Einfuhrung einer schweizerischen Unfallversicherung, Beilage II. zur Botschaft des Bundesrats vom 14.12.1889, BBl 521889 S. 825

3 PUBLIC LAW 109-41-JULY 29, 2005: PATIENT SAFETY AND QUALITY IMPROVEMENT ACT OF 2005.

4 AMA-Statement an den US-Kongress, 8. Mai 2002.

5 Bericht in Erfüllung der Postulate 12.3100 Kessler, 12.3124 Gilli und 12.3207 Steiert.
6 Bernhard Rütsche, Nadja d'Amico und Lea Schläpfer, Stärkung der Patientenrechte: internationales Soft Law und nationale Gesetze im Vergleich, Gutachten im Auftrag des BAG, 2013.

7 Rütsche op cit, S. 21: «Dass das Berichtssystem einen Lerneffekt und nicht etwa eine Bestrafung bezweckt, verdeutlicht zudem die Bestimmung, dass die meldende Person nicht einem Untersuchungs- oder Disziplinarverfahren ausgesetzt werden darf (\$201).»

8 Hanspeter Kuhn, «Congress should pass legislation to extend protections ...» "Critical Incident Reporting» und Recht, Schweiz Ärztezeitung. 2001;82.26:1394-403.

9 Hanspeter Kuhn, Georg Below: «Melden Sie keine Flugzeugunfälle auf diesem Formular!» CIRSmedical - Massnahmen für den Vertraulichkeitsschutz; Schweiz Ärztezeitung. 2003;84,26: 1399-407.

10 Hanspeter Kuhn, 10 Jahre Warten auf gesetzliche Zweckbindungsgarantie für CIRS, Editorial Schweiz Ärztezeitung. 2009;90: 495.

11 Hanspeter Kuhn, «Congress should pass legislation to extend protections ...» «Critical Incident Reporting» und Recht*, Schweiz Ärztezeitung. 2001;82,26: 1394-403.

12 Bundesgericht, Urteil vom 8.Dezember 20161B 289/2016

13 Stellungnahme CIRRNET-Ausschuss zur Beschlagnahmung einer CIRS-Meldung im Tessin, 10. August 2016 